\title{
Variabilité géographique et estimation des paramètres génétiques de la croissance en hauteur de jeunes sapins de Céphalonie (Abies cephalonica L)
}

\author{
B Fady 1 \\ avec la collaboration technique de D Vauthier ${ }^{2}$ \\ 1 Faculté des sciences et techniques de St-Jérôme, institut méditerranéen d'écologie \\ et de paléoécologie, laboratoire de botanique et écologie méditerranéenne, \\ avenue Escadrille-Normandie-Niemen, 13397 Marseille cedex 13; \\ 2 INRA, Domaine expérimental du Ruscas, RN 98, 83237 Bormes-les-Mimosas cedex, France
}

(Reçu le 23 juillet 1990; accepté le 10 février 1991)

\begin{abstract}
Résumé - La variabilité géographique de la croissance en hauteur d'Abies cephalonica a été étudiée pour 10 provenances, réparties dans 3 plantations comparatives situées dans le sud de la France. Les provenances se scindent en 3 ensembles de dynamisme différent : un groupe "Grèce orientale" de faible croissance, un groupe "Grèce continentale" de croissance intermédiaire et un groupe "Péloponnèse", de croissance plus forte. La provenance du massif du Taygète présente une hauteur totale initiale forte et une mortalité après transplantation qui la rapproche du groupe Grèce continentale, mais une croissance annuelle et une situation géographique qui la rapprochent de celles du groupe Péloponnèse. Les raisons de ces différences de croissance entre groupes pourraient être d'ordre génétique et adaptatif. Au sein du groupe Péloponnèse, les provenances du Mainalon et du Parnon ont des écovalences interannuelles et interstationnelles très faibles. Avec les provenances du Pinde étudiées dans un autre dispositif, ce sont ces provenances qui semblent le mieux convenir à des opérations de reboisements en France méditerranéenne à l'étage du chêne pubescent. La variabilité génétique des mêmes caractères de croissance a été estimée sur 3 provenances scindées en descendances maternelles individualisées et présentes sur une seule des plantations. L'héritabilité au sens strict est comprise entre 0,20 et 0,25 pour la hauteur totale à 11 ans, et entre 0,08 et 0,11 pour l'accroissement annuel cumulé de 7 à 11 ans. Si une corrélation juvénileadulte existe, ces estimations laissent supposer la possibilité d'une sélection précoce sur les caractères de croissance étudiés.
\end{abstract}

Abies cephalonica / croissance en hauteur / variabilité géographique / variabilité génétique

Summary - Geographic and genetic variability of height growth in young Abies cephalonica Loud. Geographic variability of height growth in Abies cephalonica Loud was studied in 10 provenances grown at 3 different experimental sites in southern France. Provenances could be separated into 3 different groups: first, the slow growing "Eastern Greece" group; second, the intermediate "Continental Greece" group and third, the fast growing "Peloponnesos" group. The Taygetos mountains provenance presented strong initial height growth and post-plantation mortality comparable to those of the Continental Greece group, but could be linked with the Peloponnesos group through an- 
nual increments and its geographical situation. Genetic and adaptative factors could be responsible for these differences. Within the Peloponnesos group, Menalon and Parnon provenances had the lowest site and year interactions. Along with the Pindos provenances studied in another experimental design, they seemed to be the best adapted provenances for reforestation projects in the Quercus pubescens zone in Mediterranean France. Genetic variability of height growth was estimated using family and progeny data from 3 provenances grown at 1 site. Individual heritability values were between 0.20 and 0.25 for mean total height at age $11 \mathrm{yr}$ and between 0.08 and 0.11 for cumulative annual increments from ages 7 to $11 \mathrm{yr}$. If significant juvenile-adult correlations can be found, these estimates indicate the possibility of early selection in Abies cephalonica based on height growth characteristics.

\section{Abies cephalonica / height growth / geographic variability / genetic variability}

\section{INTRODUCTION}

Abies cephalonica a été soumis en France méditerranéenne à un programme d'amélioration portant sur son adaptation et sa croissance en hauteur et en volume depuis environ 25 ans, à la suite des études écologiques réalisées dans son aire d'origine (Bassiotis, 1956 et Pauly, 1962 notamment, études enrichies depuis par celles de Panetsos, 1975 et Barbero, Quézel, 1976, entre autres). La croissance en hauteur de ce sapin est très lente dans sa phase juvénile, et il est important de pouvoir sélectionner aussi précocement que possible les génotypes les plus vigoureux.

La variabilité du caractère de croissance (hauteur totale ou pousse annuelie) donnant, en milieu homogène et dans le but d'un sélection, une idée satisfaisante de la variabilité génétique de l'espèce (Wright, 1976), ce travail a un double objectif. Le premier est d'exprimer l'allure de la variabilité géographique d'Abies cephalonica en comparaison avec celle observée dans des travaux antérieurs (Descroix, 1981; Fady, 1988) et d'individualiser les provenances les plus aptes à d'éventuels reboisements dans le sud de la France. Le second est d'estimer, en utilisant la décomposition de la variance phénotypique et les modèles de la génétique quantitative, les paramètres génétiques des caractères de croissance en hauteur.

\section{MATÉRIEL ET MÉTHODES}

\section{Matériel végétal}

Les individus étudiés se répartissent en 10 provenances (tableau I). Ces provenances sont, le cas échéant, subdivisées en descendances maternelles issues de pollinisation libre en forêt (échantillonnage aléatoire intraprovenance). Elles sont originaires de la partie méridionale de l'aire de répartition d'Abies cephalonica (fig1, d'après Barbero et Quézel, 1985) où le taux d'hybrides fixés ( $A$ borisii regis) avec $A$ alba est relativement faible (Mattfeld, 1930; Panetsos, 1975; Mitsopoulos et Panetsos, 1987; Fady et al, 1991).

Le réseau expérimental concernant ce matériel génétique est composé de 3 vergers à graines de semis : les plantations VG1 et VG2 à Saint-Lambert (Vaucluse), le site de Saint-Jurs (Alpes de Haute-Provence) et le site de Tuchan (Aude). Ces vergers ont été installés au cours de l'hiver 1980-1981 avec des plants 2-2. Leurs caractéristiques sont reprises dans le tableau II. II est à noter que sur la plantation de Tuchan, seules les provenances du Péloponnèse ( $y$ compris celles des îles de Céphalonie et d'Eubée) sont présentes.

Les paramètres génétiques ont été estimés à partir des individus du verger à graines de StLambert, qui est le seul à posséder une structure combinée provenances-descendances maternelles individualisées. Les nombres totaux d'individus mesurés figurent dans le tableau III.

Les différences importantes entre effectifs de provenances et de descendances maternelles 
Tableau I. Typologie des peuplements de sapin de Grèce d'où ont été récoltées les provenances présentes dans les plantations comparatives françaises (d'après les fiches techniques de l'Office national forestier grec).

\begin{tabular}{|c|c|c|c|c|c|c|}
\hline $\begin{array}{l}\text { Code } \\
\text { prove- } \\
\text { nance }\end{array}$ & $\begin{array}{l}\text { Localisation } \\
\text { (provenance, massif, } \\
\text { coordonnées) }\end{array}$ & $\begin{array}{l}\text { Altitude } \\
\text { exposition, } \\
\text { étage bioclimatique }\end{array}$ & $\begin{array}{l}\text { Précipi- } \\
\text { tations } \\
\text { annuelles }\end{array}$ & $\begin{array}{l}\text { Substrat* } \\
(\mathrm{mm})\end{array}$ & $\begin{array}{l}\text { Indice } \\
\text { d'aridite** }\end{array}$ & $\begin{array}{l}\text { Distance } \\
\text { aे la } \\
\operatorname{mer}(\mathrm{km})\end{array}$ \\
\hline TAYG & $\begin{array}{l}\text { Taygetos, Taygète } \\
37^{\circ} 16 \mathrm{~N}, 22^{\circ} 18 \mathrm{E}\end{array}$ & $\begin{array}{l}730-1480 \mathrm{~m} \text {, nord } \\
\text { Supra-méditerranéen }\end{array}$ & 1300 & Calcaire & 55,6 & 15 \\
\hline PNON & $\begin{array}{l}\text { Parnon, Parnon } \\
37^{\circ} 06 \mathrm{~N}, 22^{\circ} 45 \mathrm{E}\end{array}$ & $\begin{array}{l}1060-1240 \mathrm{~m} \\
\text { Supra-méditerranéen }\end{array}$ & 1200 & Calcaire & 52,6 & 15 \\
\hline VETI & $\begin{array}{l}\text { Vetina, Mainalon } \\
37^{\circ} 33 \mathrm{~N}, 22^{\circ} 15 \mathrm{E}\end{array}$ & $\begin{array}{l}950-1160 \mathrm{~m} \\
\text { Supra-méditerranéen }\end{array}$ & 1200 & Calcaire & 55,1 & 40 \\
\hline CEPH & $\begin{array}{l}\text { Cephallenia, île de Céphalonie } \\
38^{\circ} 14 \mathrm{~N}, 20^{\circ} 32 \mathrm{E}\end{array}$ & $\begin{array}{l}730-1480 \mathrm{~m} \\
\text { Supra-méditerranéen }\end{array}$ & 1100 & Calcaire & 43,3 & 3 \\
\hline ERYM & $\begin{array}{l}\text { Erymanthos, Erymanthe } \\
37^{\circ} 55 \mathrm{~N}, 21^{\circ} 50 \mathrm{E}\end{array}$ & $\begin{array}{l}980-1150 \mathrm{~m} \\
\text { Supra-méditerranéen }\end{array}$ & 1200 & Calcaire & 52,9 & 30 \\
\hline EVIA & $\begin{array}{l}\text { Evia, île d'Eubée } \\
38^{\circ} 40 \mathrm{~N}, 23^{\circ} 30 \mathrm{E}\end{array}$ & $\begin{array}{l}550-700 \mathrm{~m} \\
\text { Méso-méditerranéen }\end{array}$ & 750 & Schistes & 31,4 & 3 \\
\hline PARN & $\begin{array}{l}\text { Parnetha, Parnis } \\
38^{\circ} 11 \mathrm{~N}, 23^{\circ} 41 \mathrm{E}\end{array}$ & $\begin{array}{l}950-1050 \mathrm{~m} \\
\text { Méso-méditerranéen }\end{array}$ & 800 & Calcaire & 38,6 & 15 \\
\hline PNAS & $\begin{array}{l}\text { Parnassos, Parnasse } \\
38^{\circ} 35 \mathrm{~N}, 22^{\circ} 30 \mathrm{E}\end{array}$ & $\begin{array}{l}1050-1250 \mathrm{~m} \\
\text { Supra-méditerranéen }\end{array}$ & 1200 & Flyschs & 58,8 & 18 \\
\hline OETE & $\begin{array}{l}\text { Oete, Iti } \\
38^{\circ} 45 \mathrm{~N}, 22^{\circ} 10 \mathrm{E}\end{array}$ & $\begin{array}{l}800-1350 \mathrm{~m} \\
\text { Supra-méditerranéen }\end{array}$ & .1000 & Flyschs & 49,3 & 35 \\
\hline PNAE & $\begin{array}{l}\text { Panaetolikon, Panaetolikon } \\
38^{\circ} 43 \mathrm{~N}, 21^{\circ} 46 \mathrm{E}\end{array}$ & $\begin{array}{l}700-1000 \mathrm{~m} \\
\text { Supra-méditerranéen }\end{array}$ & 1100 & Calcaire & 49,8 & 40 \\
\hline
\end{tabular}

\footnotetext{
" Substrat d'après J Bornovas et T Rondogianni-Tsiambaou, 1983; ** Indice d'aridité de De Martonne $i=\frac{P}{T}$, où $P=$ précipitations annuelles en $\mathrm{mm}$ et $T=$ température moyenne annuelle en ${ }^{\circ} \mathrm{C}$. $T+10$
}

sont dues aux différences de faculté germinative, ainsi qu'à la mortalité en pépinière et en plantation.

\section{Méthodes}

Un comptage systématique de la mortalité après plantation a été entrepris sur les plantations de St-Lambert et de Tuchan. La variabilité du taux de reprise entre provenances a été analysée en comparant les intervalles de confiance des moyennes de provenances.

Les mesures de hauteur totale $(H T)$ et de pousse annuelle ( $P O)$ ont été réalisées à la règle télescopique graduée (précision $0,5 \mathrm{~cm}$ ) pendant l'automne 1987 et ont permis d'enregisirer rétrospectivement les valeurs de 5 années de végétation consécutives.

Le modèle général utilisé dans l'étude interprovenance est du type :

$$
x_{i j}=\mu+p_{i}+r_{i j}
$$




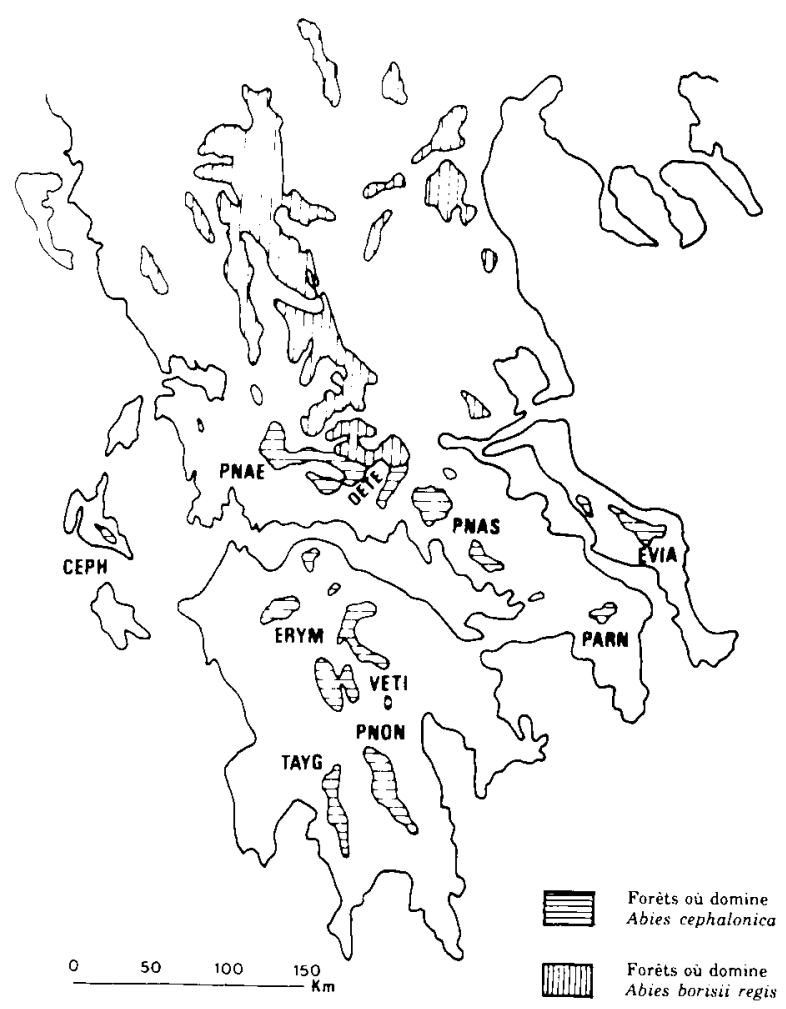

Fig 1. Situation des provenances de sapin de Céphalonie dans leur aire de répartition naturelle.

où $x_{i j}$ est le caractère analysé ( $H T$ ou $P O$ ), $\mu$ la moyenne générale, $p_{i}$ l'effet de la provenance $i$ et $r_{i j}$ l'effet résiduel. Ce modèle est obtenu après ajustement des valeurs individuelles à l'effet macro-bloc, chaque macro-bloc étant constitué de plusie' urs blocs initiaux, le nombre d'individus présents dans les blocs initiaux étant souvent trop faible pour en estimer l'effet moyen.

La signification des différences entre moyennes de familles et de provenances a été estimée par une analyse de variance nonorthogonale hiérarchique à 2 niveaux. Les moyennes ont été classées par le test de comparaison multiple de Duncan. Les moyennes de provenances ont en plus été comparées par une analyse discriminante utilisant les variables HT83 et PO84 à PO87.

À l'étude de la vigueur comparée des provenances a été ajoutée la mesure de leur stabilité spatio-temporelle, en utilisant le concept d'écovalence (Wricke, 1962). La base de cette étude de l'interaction génotype $x$ milieu est la mesure, pour la provenance $i$, de l'interactivité $W_{i}$ du caractère analysé ( $P O$ ou $H T$ ) en fonction d'un facteur du milieu : plus ce terme est important et plus la provenance considérée sera instable par rapport à son milieu.

En considérant que la valeur d'un caractère $X$ d'un individu $k$ de la provenance $i$, dans un milieu $j$, s'écrit sous la forme :

$$
X_{i j k}=\mu+P_{i}+M_{j}+(P M)_{i j}+R_{i j k}
$$

avec $\mu=$ moyenne générale, $P_{i}=$ effet de la provenance $i, M_{j}=$ effet du milieu $j$ (effet année ou effet station), $(P M)_{i j}=$ interaction provenance x milieu, et $R_{i j k}=$ erreur résiduelle, l'interactivité 


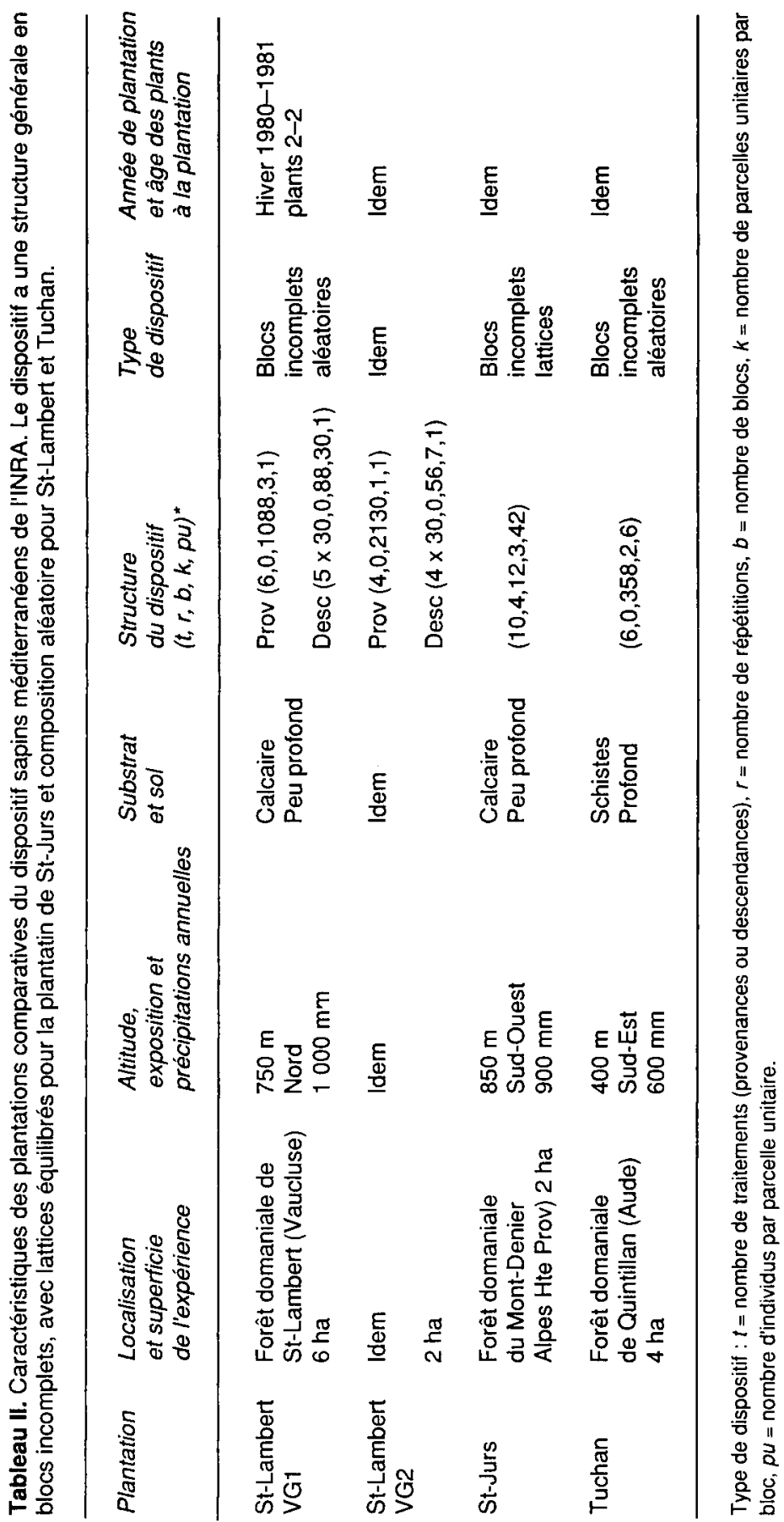


Tableau III. Effectifs par plantation des individus utilisés pour l'étude de la variabilité de la croissance en hauteur en 1987, compte tenu de la mortalité en plantation depuis l'installation en 1981.

\begin{tabular}{|c|c|c|c|c|c|c|}
\hline \multirow[t]{2}{*}{$\begin{array}{l}\text { Code } \\
\text { provenan }\end{array}$} & \multicolumn{4}{|c|}{$\begin{array}{c}\text { Variabilité inter-provenances } \\
\text { (= nombre total d'individus mesurés) }\end{array}$} & \multicolumn{2}{|c|}{ Variabilité intra-provenance } \\
\hline & $\begin{array}{l}\text { St-Lambert } \\
\quad \text { VG1 }\end{array}$ & $\begin{array}{l}\text { St-Lambert } \\
\quad \text { VG2 }\end{array}$ & St-Jurs & Tuchan & $\begin{array}{c}\text { St-Lambert } \\
\text { VG1 } \\
(i-f-i / f)^{*}\end{array}$ & $\begin{array}{c}\text { St-Lambert } \\
\text { VG2 } \\
(i-f,-i f f)\end{array}$ \\
\hline CEPH & 147 & - & 33 & 62 & - & - \\
\hline ERYM & 884 & - & 95 & 519 & $(470-20-23,5)$ & _- \\
\hline VETI & 1166 & - & 79 & 363 & $(631-27-23,4)$ & - \\
\hline PNON & 653 & - & 74 & 187 & $(200-14-14,3)$ & - \\
\hline TAYG & 25 & - & 88 & 48 & $(25-3-8,3)$ & - \\
\hline EVIA & 67 & _- & 44 & 145 & $(67-17-3,9)$ & - \\
\hline PARN & - & 467 & 23 & - & - & $(10-2-5)$ \\
\hline PNAS & - & 74 & 50 & - & - & $(74-9-8,2)$ \\
\hline OETE & - & 439 & 60 & - & _ & $56-6-9,3)$ \\
\hline PNAE & - & 256 & 55 & - & - & $(22-4-5,5)$ \\
\hline
\end{tabular}

Variabilité intra-provenance : $i=$ nombre total d'individus mesurés, $f=$ nombre de familles observées, $i / f=$ nombre moyen d'individus par famille.

de la provenance $i$ (inverse mathématique de l'écovalence) prend la forme:

$$
W_{i}=\sum_{j}\left[\left(X_{i j}-X_{i} . .\right)-\left(X_{. j}-X_{\ldots .}\right)\right]^{2}
$$

Dans l'analyse suivante, ce seront les écovalences relatives (Baradat, 1989, 69-74) qui seront utilisées :

$$
W_{r i}=100 \overline{\Sigma_{i} W_{i}}
$$

Le modèle général utilisé dans l'étude intraprovenance est du type :

$$
x_{i j k}=\mu_{i}+f_{i j}+r_{i j k}
$$

où $x_{i j k}$ est le caractère analysé (HT ou $P O$ ), $\mu_{i}$ la moyenne de la provenance $i, f_{i j}$ l'effet de la famille $j$ dans la provenance $i$ et $r_{i j k}$ l'effet résiduel.

Les paramètres génétiques ont été calculés sur l'ensemble des individus, en supposant l'homogénéité des matrices de variance/covariance résiduelles et familiales pour les différentes provenances. II existe probablement une consanguinité importante en forêt naturelle. Le coefficient de consanguinité $(F)$ a pu être évalué à
0,6 à partir des fréquences des individus riches ou pauvres en un terpène (limonène) dans un peuplement naturel d'Abies alba (Moreau, 1989) et semble pouvoir atteindre, en faisant une estimation indirecte sur le même terpène, une valeur maximale de 0,7 pour les provenances d'Abies cephalonica étudiées (Fady, 1990a). Une étude isoenzymatique portant sur un grand nombre de loci chez Abies lasiocarpa (Shea, 1990) montre que le coefficient moyen de consanguinité est de 0,341. L'appareniement entre arbres mères n'a pas été envisagé et sera supposé nul. Ainsi, en utilisant la covariance entre apparentés qui permet de faire correspondre effets génétiques additifs et effets famille, et en supposant l'absence d'épistasie et une représentation exclusive de demi-frères dans les descendances maternelles, l'héritabilité au sens strict (Falconer, 1974) s'exprime, pour des valeurs moyennes de $F$ variant entre 0,3 et 0,6 sous la forme:

$$
h^{2}=\frac{4}{1+F} \quad \frac{\sigma^{2 f}}{\sigma^{2} f+\sigma^{2} r}, \text { d'où }
$$




$$
2,5 \frac{\sigma^{2} f}{\sigma^{2} f+\sigma^{2} r}<h^{2}<3,1 \quad \frac{\sigma^{2} f}{\sigma^{2} f+\sigma^{2} r}
$$

où $\sigma^{2} f$ représente la variance d'un caractère au niveau familial et $\sigma^{2} r$ représente la variance résiduelle.

Les coefficients de prédiction génétique (Baradat, 1976) entre 2 caractères $x_{1}$ et $x_{2}$ estiment l'efficacité de la prédiction du gain génétique réalisé sur un caractère $x_{1}$ en faisant une sélection indirecte sur un caractère $x_{2}$. Ils s'expriment sous la forme:

$$
\operatorname{CPG}\left(x_{1}, x_{2}\right)=\frac{\operatorname{Cov}_{A}\left(x_{1}, x_{2}\right)}{\sigma_{P}\left(x_{1}\right) \sigma_{P}\left(x_{2}\right)}
$$

où $\operatorname{Cov}_{A}$ représente la covariance génétique additive et $\sigma_{P}$ l'écart type phénotypique.

Étant donnés les problèmes de faibles effectifs d'individus par familles et de familles par provenance, seules les familles des provenances ERYM, VETI et PNON de VG1 à St-Lambert ont été retenues dans le calcul des paramètres génétiques.

Les erreurs standard des héritabilités et des coefficients de corrélation génétique et phénotypique (Mode, Robinson, 1959) ont permis de calculer un intervalle de confiance de ces estimations.

\section{RÉSULTATS}

\section{Mortalité}

Le tableau II indique que le nombre initial de familles par provenance était de 30 . À la suite d'une prolifération de Phytophthora cinammomi en pépinière, et compte tenu de la mortalité après transplantation, le nombre final de familles et d'individus survivants en 1987 à St-Lambert est repris dans le tableau III.

À St-Lambert, les taux de mortalité varient en fonction des provenances. Même en ne tenant pas compte d'EVIA, qui a une mortalité significativement plus élevée que celle des autres provenances (tableau IV), l'écart entre provenances varie du simple au double (PNAE $=22,3 \%$ et $\mathrm{CEPH}=$ $53,0 \%)$.

Le coefficient de corrélation linéaire entre pourcentage de mortalité observé en 1987 et conditions écologiques du milieu d'origine (TAYG non compris, qui présente un effectif trop faible) montre des corrélations significatives avec la température moyenne annuelle $\left(r=0,712^{\star}\right.$, significatif à $5 \%)$ et l'éloignement de la mer ( $r=-$ $0,822^{\star \star}$, significatif à $\left.1 \%\right)$. Étant donné le petit nombre de couples de points utilisables, cette corrélation est sans doute à considérer avec précaution.

Chaque année n'a pas une importance égale dans la mortalité des provenances : ainsi, l'année qui suit directement l'année de plantation présente un taux de mortalité très élevé, en général supérieur à $50 \%$ de la mortalité totale (sauf PNAE) au moment des mesures (1987), et jusqu'à $77,5 \%$ (PNON, tableau IV). Le taux de mortalité après plantation est plus élevé chez les provenances du verger VG1 (supérieur à $61 \%$, sauf TAYG $51 \%$ ) que chez celles de VG2 (entre 46 et $56 \%$ ).

La mortalité moyenne à St-Lambert $(36,0 \%)$ n'est pas significativement différente de celle de Tuchan $(40,7 \%)$. La mortalité à St-Jurs n'a pas été étudiée.

\section{Variabilité intra-provenance}

Tous les tests $F$ de l'effet famille (modèle aléatoire) sur VG1 à St-Lambert sont significatifs à $1 \%$, sauf pour la pousse annuelle 1985 ( $P O 85$, significatif à $5 \%$ ). Le tableau $V$ indique l'allure de la variabilité interfamilles des provenances de VG1 pour le caractère $P O 87$. L'amplitude de la variabilité est pratiquement la même pour toutes les provenances. Le coefficient de variation relativement plus élevé d'EVIA pourrait 
Tableau IV. Évolution de la mortalité dans le temps sur l'ensemble des provenances du verger à graines de St-Lambert. ND : non disponible.

\begin{tabular}{|c|c|c|c|c|}
\hline $\begin{array}{l}\text { Provenances et } \\
\text { verger d'origine }\end{array}$ & $\begin{array}{c}\% \text { total de mortalité } \\
\text { entre la plantation } \\
\text { et } 1987\end{array}$ & $\begin{array}{c}\text { Mortalité en } 1981 \\
\text { en \% de la } \\
\text { mortalité totale }\end{array}$ & $\begin{array}{c}\text { Mortalité en } 1982 \\
\text { en \% de la } \\
\text { mortalité totale }\end{array}$ & $\begin{array}{c}\text { Mortalité après } 1981 \\
\text { en \% de la } \\
\text { mortalité totale }\end{array}$ \\
\hline CEPH (VG1) & 53,00 & 85,40 & ND & 14,60 \\
\hline ERYM (VG1) & 23,90 & 68,10 & 13,10 & 31,90 \\
\hline VETI (VG1) & 29,30 & 61,40 & 16,00 & 38,60 \\
\hline PNON (VG1) & 43,25 & 77,50 & 8,90 & 22,50 \\
\hline TAYG (VG1) & 37,40 & 51,00 & 27,80 & 49,00 \\
\hline EVIA (VG1) & $62,85^{* *}$ & 67,10 & 18.90 & 32,90 \\
\hline PARN (VG2) & 26,27 & 53,40 & ND & 46,60 \\
\hline PNAS (VG2) & 33,77 & 54,90 & ND & 45,10 \\
\hline OETE (VG2) & 27,56 & 56,60 & ND & 43,40 \\
\hline PNAE (VG2) & 22,30 & 46,80 & ND & 53,20 \\
\hline Moyenne & 36,00 & 62,20 & - & - \\
\hline
\end{tabular}

* Provenance significativement différente au seuil de $1 \%$.

Tableau V. Coefficients de variation interfamilles de la pousse annuelle 1987 pour chaque provenance à St-Lambert.

\begin{tabular}{lcc}
\hline Provenances & $\begin{array}{c}\text { Nombre de } \\
\text { familles }\end{array}$ & $\begin{array}{c}\text { Coefficient de } \\
\text { variation }\end{array}$ \\
\hline ERYM & 20 & 0,16 \\
VETI & 27 & 0,15 \\
PNON & 14 & 0,20 \\
EVIA & 17 & 0,45 \\
PNAS & 9 & 0,20 \\
Moyenne & & 0,15 \\
\hline
\end{tabular}

être l'effet d'une surestimation due à son faible nombre d'individus par famille, plutôt que la manifestation de familles plus variables.

Les héritabilités $\left(h^{2}\right)$ sont semblables d'une année sur l'autre, et plus fortes pour HT que pour $P O$ (tableau VI). Les coeffi- cients de prédiction génétique ( $C P G$ ) de la hauteur totale 1987 (HT87) par les hauteurs totales des années précédentes ne montrent pas d'augmentation significative avec l'âge, et sont pratiquement identiques aux $h^{2}$. En revanche, les CPG de HT87 par les $P O$ sont toujours plus élevés que les $h^{2}$ des $P O$.

Les corrélations entre caractères (tableau VII) sont comparables sur les 2 plantations. Les corrélations HT-PO sont toujours significatives à $1 \%$, mais varient selon l'année considérée. La corrélation la plus forte est obtenue entre $H T$ et $P O$ cumul, traduisant l'intérêt de cette variable dans la prédiction de la hauteur totale. L'existence d'un effet année se retrouve dans l'apparition de corrélations $P O-P O$, dont le seuil de signification est très variable.

La part de variabilité due à la provenance dans la variance génétique additive totale est comprise entre 10 et $38 \%$ pour 
Tableau VI. Héritabilités au sens strict $\left(h^{2}\right)$ des caractères de hauteur totale $(H T)$ et de pousse annuelle $(P O)$ entre 1983 et 1987 en fonction de deux valeurs extrêmes du coefficient de consanguinité (F) et coefficient de prédiction génétique (CPG) de la hauteur totale 1987 (HT87) par différents caractères au niveau intra-provenance sur les plantations du verger à graines de St-Lambert.

\begin{tabular}{llcc}
\hline Caractères & $\begin{array}{c}h^{2} \\
F=0,6\end{array}$ & $\begin{array}{c}h^{2} \\
F=0,3\end{array}$ & $\begin{array}{c}C P G H T 87 \\
(0,6>F>0,3)\end{array}$ \\
\hline HT83 & $0,19^{* *}$ & $0,24^{* *}$ & $0,19-0,24^{* *}$ \\
HT84 & $0,22^{* *}$ & $0,28^{* *}$ & $0,20-0,25^{* *}$ \\
HT85 & $0,20^{* *}$ & $0,25^{* *}$ & $0,19-0,24^{* *}$ \\
HT86 & $0,20^{* *}$ & $0,25^{* *}$ & $0,19-0,24^{* *}$ \\
PO84 & $0,20^{\star *}$ & $0,25^{\star *}$ & $h^{2}$ \\
PO85 & $0,06^{* *}$ & $0,08^{* *}$ & $0,11-0,13^{*}$ \\
PO86 & $0,04^{*}$ & $0,05^{*}$ & $0,07-0,10^{*}$ \\
PO87 & $0,08^{* *}$ & $0,11^{* *}$ & $0,11-0,13^{*}$ \\
PO cumul (84-87) & $0,13^{\star *}$ & $0,16^{* *}$ & $0,09-0,12^{*}$ \\
& $0,08^{\star *}$ & $0,11^{* *}$ & $0,13-0,16^{*}$ \\
\hline
\end{tabular}

* significatif à $5 \%$, (au seuil de $5 \%$, l'intervalle de confiance des estimations des paramètres génétiques ne recoupe pas la valeur 0 ); " significatif à $1 \%$.

Tableau VII. Corrélations génétiques et phénotypiques entre caractères de croissance (hauteur totale, $H T$ et pousse annuelle, $P O$ ) sur les vergers VG1 et VG2 de la plantation de St-Lambert.

\begin{tabular}{lcccc}
\hline & \multicolumn{2}{c}{ VG1 } & \multicolumn{2}{c}{ VG2 } \\
Caractères & Phénotypique & Génétique & Phénotypique & Génétique \\
& & & & \\
HT87-HT84 & $0,81^{* *}$ & $0,94^{* *}$ & $0,82^{* *}$ & $0,91^{* *}$ \\
HT87-HT83 & $0,68^{* *}$ & $0,99^{* *}$ & $0,67^{* *}$ & $0,78^{* *}$ \\
HT87-PO87 & $0,52^{* *}$ & $0,58^{* *}$ & $0,63^{* *}$ & $0,78^{* *}$ \\
HT87-PO86 & $0,71^{* *}$ & $0,92^{* *}$ & $0,66^{* *}$ & $0,63^{* *}$ \\
HT87-PO85 & $0,69^{* *}$ & $0,76^{* *}$ & $0,62^{* *}$ & $0,98^{* *}$ \\
HT87-PO84 & $0,67^{* *}$ & $0,95^{* *}$ & $0,62^{* *}$ & $0,76^{* *}$ \\
HT87-POCumul & $0,86^{* *}$ & $0,99^{* *}$ & $0,79^{* *}$ & $0,92^{* *}$ \\
PO87-PO86 & $0,60^{* *}$ & $1,01^{* *}$ & $0,70^{* *}$ & $\mathrm{NS}$ \\
PO87-PO85 & $0,29^{* *}$ & $\mathrm{NS}$ & $0,33^{* *}$ & $\mathrm{NS}$ \\
PO87-PO84 & $\mathrm{NS}$ & $\mathrm{NS}$ & $\mathrm{NS}$ & $\mathrm{NS}$ \\
\hline
\end{tabular}

NS : corrélation non significativement differente de $0 .{ }^{*}$ significatif à $5 \%$; ${ }^{* *}$ significatif à $1 \%$ (au seuil de $1 \%$, l'intervalle de confiance des coefficients de corrélation ne recoupe pas la valeur $(0)$. 
les $P O$ et entre 4 et $17 \%$ pour les $H T$ selon l'année considérée (tableau VIII).

\section{Variabilité inter-provenances}

St-Lambert est la plantation qui a donné les résultats les plus précis. Les 2 sites VG1 et VG2 ont été réunis en une seule plantation pour cette étude : ils ne présentent aucune différence pédo-climatique notable, et la variance résiduelle de chaque caractère est comparable pour les 2 plantations.

Les tests $F$ de l'effet provenance sont significatifs à $1 \%$, sauf pour $P 086$ et $P 087$ (non significatifs). Les classements de $H T$ présentent quelques changements avec l'âge (fig 2). Si PARN et EVIA sont toujours en fin de classement, et parfois de manière significative, au groupe de tête de 1983, composé essentiellement de TAYG et des provenances de Grèce continentale (PNAE, OETE, PNAS), s'ajoute en 1987 un groupe contenant les provenances du Péloponnèse (VETI, PNON, CEPH).

Les classements de $P O$ (fig 3 ) montrent que l'augmentation de vigueur des provenances du Péloponnèse entre 1983 et 1987 est due à une croissance annuelle plus importante que celles des autres provenances. PARN et EVIA ont systématiquement les $P O$ les plus faibles.

L'analyse discriminante (fig 4) permet de distinguer trois groupes de prove- nances dans le plan factoriel 1-2 (taux d'inertie de l'axe $1=55,1 \%$ et taux d'inertie de l'axe $2=38,9 \%$, soit un taux cumulé de $94 \%$ ); l'axe 1 va dans le sens de valeurs élevées de $P O 84$ et $P O 85$ et l'axe 2 dans le sens d'une valeur élevée de HT87. Ces 2 axes opposent donc les provenances ayant une valeur initiale de $H T$ élevée à celles dont la croissance annuelle est importante les années suivantes:

- le Péloponnèse (provenances VETI, ERYM, PNON et CEPH), caractérisé par des $P O$ fortes et une hauteur totale initiale (HT83) moyenne;

- la Grèce continentale (PNAS, PNAE et OETE), aux croissances annuelles moyennes mais à la hauteur totale initiale la plus forte;

- les provenances PARN et EVIA, peu vigoureuses. Ce groupe est toujours significativement distant au seuil de $5 \%$ des 2 autres.

La provenance TAYG n'est pas significativement différente des 2 premiers groupes : comme les provenances de Grèce continentale, sa hauteur initiale est la plus élevée et comme les provenances du Péloponnèse, sa croissance annuelle est la plus forte.

Les résultats obtenus sur les 2 autres plantations sont souvent moins significatifs. Les tests $F$ sur les provenances sont significatifs à $1 \%$ pour tous les caractères, sauf pour les pousses 1986 et 1987 . Cependant, le classement des performances

Tableau VIII. Pourcentage de variance due au facteur provenance dans la variance génétique additive totale pour les caractères de hauteur totale $(H T)$ et de pousse annuelies (PO) à S-Lambert (Vaucluse).

\begin{tabular}{llllllllll} 
HT83 & HT84 & HT85 & HT86 & HT87 & PO84 & PO85 & PO86 & PO87 & PO84-87 \\
\hline 4,6 & 9,6 & 14,2 & 15,9 & 17,0 & 35,6 & 38,2 & 21,1 & 9,5 & 32,4 \\
\hline
\end{tabular}


HT 1983

HT 1985

HT 1987

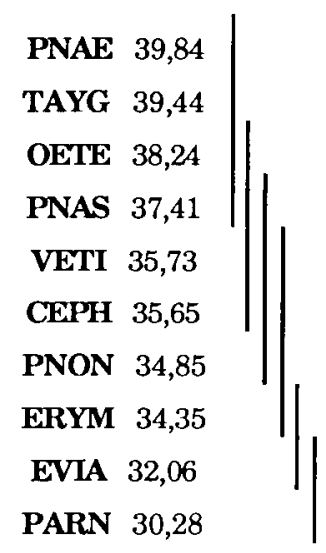

Moyenne $=35,19$

\begin{tabular}{cc|} 
TAYG & 59,32 \\
VETI & 55,72 \\
PNAE & 55,34 \\
CEPH & 54,91 \\
PNON & 54,64 \\
OETE & 54,25 \\
PNAS & 53,22 \\
ERYM & 52,60 \\
EVIA & 45,70 \\
PARN & 44,13
\end{tabular}

Moyenne $=53,20$ \begin{tabular}{cc|} 
TAYG & 74,20 \\
OETE & 73,56 \\
VETI & 70,77 \\
PNON & 69,08 \\
CEPH & 68,95 \\
PNAE & 67,30 \\
ERYM & 66,98 \\
PNAS & 65,35 \\
EVIA & 55,56 \\
PARN & 54,31
\end{tabular}

Moyenne $=67,56$

Fig 2. Comparaison de quelques classements de hauteurs totales $(H T)$ en $\mathrm{cm}$ sur la plantation de StLambert (Vaucluse). Test de Duncan au seuil de $5 \%$ (toute provenance non reliée à une autre par un barre verticale lui est significativement différente pour le caractère considéré).

des provenances est en général comparable à celui de St-Lambert. Ainsi, à StJurs, PARN est une provenance significativement plus petite que les autres (fig 5), et à Tuchan (où la Grèce continentale n'est pas représentée) cette place revient à EVIA (fig 6). À St-Jurs, le regroupement des provenances ne correspond pas exactement à une réalité géographique. II est à noter qu'au moment des mesures, cette plantation était envahie par une végétation arbustive, constituée principalement de genêts, qui a pu nuire à la mise en évidence de la variabilité géographique.

La stabilité des caractères de croissance (tableaux IX et $X$ ) a été étudiée en fonction de l'année (influence du climat et de l'évolution avec l'âge) et de la station. Les 3 stations présentent des fertilités différentes (tableau XI). Les tests $F$ d'interaction provenance $x$ année ne sont significa- tifs (seuil de $5 \%$ ) que pour VG1, et les tests $\mathrm{F}$ d'interaction provenance $\mathrm{x}$ station ne sont significatifs (seuil de $5 \%$ ) que pour les provenances du Péloponnèse, les seules à être représentées dans les 3 stations. VETI et PNON sont les provenances les plus stables, aussi bien vis-à-vis de l'âge et du climat que de la station. CEPH et ERYM présentent une stabilité interstationnelle importante. EVIA et TAYG tout particulì̀rement se révèlent instables.

II faut noter que sur les 3 stations, la croissance annuelle a décru entre 1984 et 1987. Cette évolution est contraire à celle qui est attendue à cet âge (Fady, 1988); la sécheresse répétée des mois de mai et juin des années d'étude est sans doute à mettre en cause dans l'expression de ce phénomène.

L'étude de la liaison entre les performances des provenances (HT87, St- 


$\begin{array}{cc}\text { PO } 1984 \\ \text { TAYG } & 10,52 \\ \text { VETI } & 10,15 \\ \text { PNON } & 10,10 \\ \text { CEPH } & 9,68 \\ \text { ERYM } & 9,06 \\ \text { PNAE } & 8,69 \\ \text { OETE } & 8,68 \\ \text { PNAS } & 8,55 \\ \text { PARN } & 7,67 \\ \text { EVIA } & 7,25\end{array}$

Moyenne $=9,30$

\begin{tabular}{cc|} 
PO 1985 \\
VETI & 9,84 \\
PNON & 9,69 \\
CEPH & 9,48 \\
TAYG & 9,36 \\
ERYM & 9,20 \\
OETE & 7,33 \\
PNAS & 7,26 \\
PNAE & 6,81 \\
EVIA & 6,39 \\
PARN & 6,19
\end{tabular}

Moyenne $=8,70$

Fig 3. Comparaison de quelques classements de pousses annuelles $(P O)$ en $\mathrm{cm}$ sur la plantation de St-Lambert (Vaucluse). Test de Duncan au seuil de $5 \%$ (toute provenance non reliée à une autre par une barre verticale lui est significativement différente pour le caractère considéré).

Tableau IX. Comparaison des provenances de VG1 St-Lambert sur la base de la moyenne de leur pousse annuelle ( $P O$ ) et de leur écovalence interannuelle (notable si supérieure à 20) pour les 5 années d'étude.

\begin{tabular}{lcc}
\hline Provenances & $\begin{array}{c}\text { PO moyenne } \\
\text { en cm }\end{array}$ & $\begin{array}{c}\text { Écovalence } \\
\text { interannuelle } \\
\text { relative }\end{array}$ \\
\hline & & \\
PNON & 9,09 & 0,12 \\
VETI & 8,90 & 10,41 \\
TAYG & 8,69 & 38,92 \\
ERYM & 8,24 & 22,84 \\
EVIA & 5,88 & 27,72 \\
& & \\
\hline
\end{tabular}

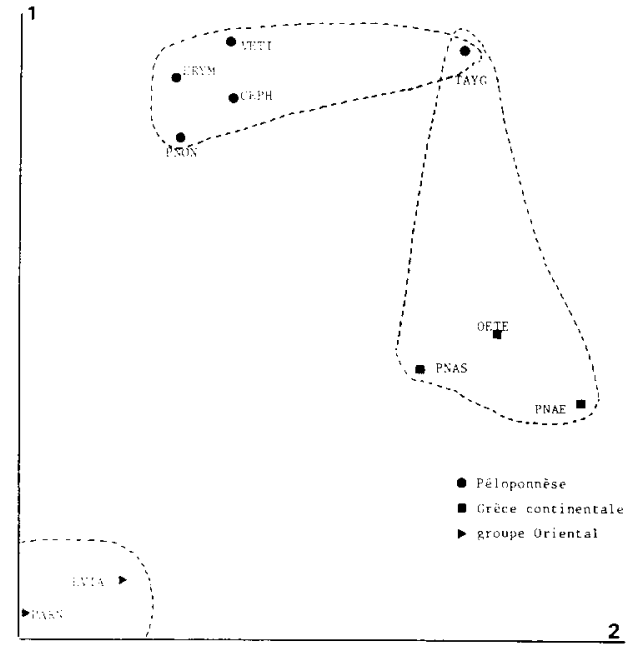

Fig 4. Distribution des provenances de sapin de Grèce de St-Lambert dans le plan factoriel 1-2 de l'analyse factorielle discriminante réalisée sur la hauteur totale 1983 et les pousses annuelles de 1984 à 1987. Les provenances regroupées dans un même ensemble sont significativement différentes de celles d'un autre ensemble au seuil de $5 \%$.

Tableau X. Comparaison des provenances du Péloponnèse de St-Lambert, St-Jurs et Tuchan sur la base de leur hauteur totale moyenne (HT) en 1987 et de leur écovalence interstationnelle relative (notable si supérieure à 16,67 ).

$\begin{array}{cc}\text { Provenances } & \text { HT } 1987 \text { Écovalence } \\ \text { en } \mathrm{cm} \quad \begin{array}{c}\text { interstationnelle } \\ \text { relative }\end{array}\end{array}$

$\begin{array}{lll}\text { VETI } & 63,41 & 4,28 \\ \text { CEPH } & 63,23 & 9,94 \\ \text { PNON } & 62,31 & 0,01 \\ \text { TAYG } & 62,89 & 46,35 \\ \text { ERYM } & 60,60 & 1,55 \\ \text { EVIA } & 53,45 & 37,88\end{array}$




\begin{tabular}{cc|cc|} 
TAYG & 20,80 & PNON & 50,93 \\
PNON & 20,60 & PNAE & 48,45 \\
VETI & 20,47 & PNAS & 48,44 \\
PNAS & 19,93 & TAYG & 48,42 \\
PNAE & 19,90 & CEPH & 48,19 \\
ERYM & 19,22 & VETI & 47,93 \\
EVIA & 18,26 & ERYM & 46,42 \\
CEPH & 18,09 & EVIA & 44,03 \\
OETE & 17,92 & OEIE & 43,62 \\
PARN & 11,68 & PARN & 35,16
\end{tabular}

Moyenne $=19,36 \quad$ Moyenne $=47,03$

Fig 5. Comparaison de quelques classements de hauteurs totales $(H T)$ et pousses annuelles $(P O)$ en $\mathrm{cm}$ sur la plantation de St-Jurs (Alpes de Haute-Provence). Test de Duncan au seuil de $5 \%$ (toute provenace non reliée à une autre par une barre verticale lui est significativement diférente pour le caractère considéré).

Tableau XI. Comparaison de la fertilité (mesurée par la hauteur totale en 1987, HT87) et de la dynamique de croissance (mesurée par le cumul des pousses annuelles entre 1984 et 1987, PO84-87) des 3 sites expérimentaux.

\section{Plantations $\quad \mathrm{HT} 87$ en cm PO84-87 en cm}

\begin{tabular}{lll}
\hline & & \\
St-Lambert & 67,56 & 32,26 \\
Tuchan & 54,77 & 24,51 \\
St-Jurs & 47,03 & 19,36 \\
\hline
\end{tabular}

Lambert) et les caractéristiques écologiques de leur lieu d'origine montre que les corrélations sont significatives à $5 \%$ pour la pluviométrie annuelle $(r=0,769)$, l'indice d'aridité de De Martonne $(r=0,715)$ et la

\begin{tabular}{c||cc|} 
VETI 25,59 & CEPH 56,52 \\
PNON 25,35 & PNON 55,94 \\
CEPH 24,60 & ERYM 55,49 \\
TAYG 24,50 & VETI 55,31 \\
ERYM 24,24 & TAYG 54,42 \\
EVIA 21,66 & EVIA 48,68 \\
Moyenne $=24,51$ & Moyenne $=54,77$
\end{tabular}

Fig 6. Comparaison de quelques classements de hauteurs totales (HT) et pousses annuelles $(P O)$ en $\mathrm{cm}$ sur la plantation de Tuchan (Aude). Test de Duncan au seuil de 5\% (toute provenance non reliée à une autre par une barre verticale lui est significativement différente pour le caractère considéré).

longitude $(r=-0,672)$. Ces corrélations sont elles aussi à prendre avec précautions, étant calculées à partir de peu de points.

\section{DISCUSSON}

Les provenances de sapin de Céphalonie ont une mortalité forte (entre 22 et $53 \%$ des individus); elle s'élève jusqu'à $63 \%$ chez la provenance EVIA. À titre d'exemple, Portefaix (1989) notait pour Pinus nigra laricio une mortalité comprise entre 16 et $22 \%$ en plantation située hors de la zone climatique méditerranéenne. II apparaît que la mortalité est plus élevée chez les provenances issues des régions de l'aire de répartition les plus chaudes et les plus proches de la mer, traduisant sans doute pour les jeunes plants des conditions d'installation très difficiles. 
La cause de cette mortalité importante doit certainement être recherchée dans l'existence d'une crise de transplantation, aggravée par les conditions rigoureuses du climat méditerranéen; le fait que la plus grosse part de cette mortalité intervienne l'année qui suit la plantation en est une indication. II n'est cependant pas possible d'ignorer l'existence d'un prolongement possible de l'importante mortalité d'origine fongique (Phytophthora) observée en pépinière.

Le stress qui s'exprime l'année suivant la transplantation semble plus important sur les provenances du Péloponnèse ( $y$ compris EVIA et CEPH) de la plantation VG1 ( 61 à $85 \%$ de la mortalité totale) que sur celles de Grèce continentale de VG2 (46 à $56 \%$ de la mortalité totale), alors que l'inverse se produit les années suivantes. Étant donnée la relative similitude de ces 2 plantations, ceci pourrait indiquer que les plants du Péloponnèse, à l'exception de TAYG, souffrent d'un stress relativement plus court, et qu'une fois celui-ci surmonté, leur installation est mieux assurée. La crise de transplantation semble plus longue pour les provenances de Grèce continentale, probablement moins bien adaptées au climat méditerranéen.

L'importance du stress de transplantation, sans doute accrue en région méditerranéenne, inciterait à comparer très soigneusement les avantages et les désavantages de différentes méthodes de plantation, notamment la méthode à racines nues, telle qu'elle a été pratiquée à St-Lambert, par opposition à la méthode en conteneurs.

L'existence d'effets maternels (grosseur de la graine, épaisseur des téguments, nature des réserves, etc) n'a pas pu être testée, faute d'informations nécessaires, sur les graines au niveau descendance. Fady et al (1991) avaient montré pour des provenances très comparables de la même espèce, une absence de corrélation au niveau provenance entre croissance juvénile et grosseur des graines.

La valeur relative aux pousses annuelles la moins affectée par les variations environnementales, et qui possède le meilleur pouvoir prédicteur de la hauteur totale est l'accroissement cumulé de 7 à 11 ans. La valeur de hauteur totale dont la variation est la moins susceptible d'être entachée d'effets maternels et d'un «bruit de fond" $\left(\sigma^{2} r\right)$ lié à la crise de transplantation (Burdon et Sweet, 1976) est la hauteur totale à 11 ans, la dernière mesurée.

L'héritabilité de l'accroissement cumulé de la pousse annuelle entre 7 et 11 ans est comprise entre 0,08 et 0,11 , et l'héritabilité de la hauteur totale à 11 ans se situe entre 0,20 et 0,25 . Si les corrélations juvénile-adulte se révèlent significatives, une sélection précoce de ces caractères de croissance en hauteur peut être envisagée.

Aucune diminution sensible de l'héritabilité n'est observable entre 1983 (7 années de végétation) et 1987 (11 années de végétation), à la différence de ce qu'observaient au même âge Namkoong et al (1972) sur Pseudotsuga menziesii et Namkoong et Conkle (1976) sur Pinus ponderosa. Cette stabilité de l'héritabilité pourrait être le reflet du maintient d'une stratégie de croissance de type juvénile pendant cette période, stratégie que le sapin de Céphalonie garde au moins jusqu'à 15-18 ans en plantation (Fady, 1988). Celle-ci se caractérise par des pousses annuelles toujours très faibles (en moyenne entre 5 et 9 $\mathrm{cm}$ pour les années étudiées). Étant donnée la durée de cette période de croissance réduite, il semble aussi souhaitable de pouvoir déterminer des prédicteurs précoces de la croissance en hauteur : la composante morphogénétique "nombre d'entre-nœuds initiés" pourrait être intéressante à cet égard (Fady, 1990b). 
La part de variabilité due au facteur provenance dans la variabilité génétique totale est inférieure à celle qui est due à la variabilité familiale et individuelle, mais elle est suffisante pour qu'apparaissent des différences significatives entre provenances. La variablité inter-provenances est comparable d'une plantation à l'autre, bien que les 3 stations soient de fertilités (estimées par dernière mesure de hauteur totale) très différentes. De toute évidence, la plantation de St-Lambert est celle qui convient le mieux au sapin de Céphalonie. C'est elle également qui permet la meilleure expression de la variabilité inter-provenances. Mis à part les problèmes de concurrence avec la végétation naturelle, comme c'est le cas à St-Jurs, les précipitations annuelles plus élevées des monts du Vaucluse comparées à celles des Corbières, sont sans doute en partie responsables de cette difféence de fertilité. Trois groupes de provenances apparaissent :

1) Les provenances PARN et EVIA, formant le groupe "Grèce orientale", très différent du reste des provenances et caractérisé par une faible croissance. L'individualisation de ce groupe se retrouve tout aussi nettement en analysant la variation géographique de caractères tels que la phénologie du débourrement végétatif et la composition terpénique (Fady, 1990a). Cette concordance multi-caractère tendrait à prouver l'originalité génétique de ce groupe, probablement par l'action combinée de flux génétiques en provenance d'autres sapins méditerranéens (races sud-orientales d'Abies alba et Abies bornmuelleriana principalement) au cours des temps géologiques et d'une dérive génétique plus récente (disjonction géographique des peuplements de sapins au cours de l'Holocène), sans doute due à des conditions écologiques très xérothermiques et à une forte pression humaine.

2) Les provenances PNAS, OETE et PNAE, formant le groupe "Grèce continen- tale", de croissance annuelle moyenne, mais dont la hauteur totale initiale (au début des mesures en 1983) était élevée : seule la provenance OETE présente certaines années un accroissement annuel (1986 et 1987) non significativement différent de celui des provenances du groupe suivant, qui explique sa hauteur totale importante en 1987.

3) Les provenances VETI, PNON, CEPH et ERYM, formant le groupe "Péloponnèse", de forte croissance, auquel se rattache la provenance TAYG, bien qu'elle puisse présenter des liens significatifs, soit avec la provenance EVIA à Tuchan (hauteur totale et croissance annuelle faibles), soit avec le groupe "Grèce continentale" à St-Lambert et à St-Jurs (forte hauteur totale initiale à 6 ans) : cette provenance du Taygète s'individualise de la sorte pour d'autres caractères, notamment sa composition terpénique, ce qui donne tout lieu de croire à l'existence d'une histoire génétique particulière, soit par dérive génétique à partir de l'ensemble "Péloponnèse", soit parce que les flux géniques provenant de la migration d'Abies alba au cours des glaciations au Quaternaire ne sont pas parvenus jusque dans les refuges glaciaires de l'Extrême-Sud du Péloponnèse, où se trouvait vraisemblablement cette provenance. La distinction entre Grèce continentale et Péloponnèse n'est pas nettement visible à St-Jurs, où chacune des provenances du Péloponnèse se rattache de manière particulière à l'ensemble bien marqué des provenances de Grèce continentale. Elle n'était de même pas toujours visible à St-Lambert pour des caractères comme le débourrement et la composition terpénique (Fady, 1990a). Les migrations quaternaires d'Abies alba sont sans doute responsables d'une certaine uniformisation des pools génétiques des individus présents dans les refuges glaciaires; la recolonisation Holocène à partir d'un petit nombre d'individus 
et la disjonction géographique récente des massifs du Péloponnèse explique tout à la fois une certaine ressemblance, mais aussi un comportement distinct par rapport aux provenances de Grèce continentale, pour lesquelles Panetsos (communication personnelle) estime, sur la base de la pubescence du rameau de l'année, que la présence d'Abies alba était beaucoup plus marquée.

L'ensemble des analyses montre que les provenances du Péloponnèse, qui sont à la fois les plus vigoureuses et les plus stables, sont celles du Mainalon (VETI) et du Parnon (PNON); en matière de reboisement, elles semblent devoir représenter un choix sûr. Cela confirme les études précédentes (Descroix, 1981; Fady, 1988; Fady et al, 1991) qui avaient montré l'intérêt des provenances du Mainalon, ainsi que celles du Pinde, qui n'ont pas été envisagées dans cette étude. Les autres provenances du Péloponnèse sont plus instables, tout particulièrement celle du Taygète. Les provenances de Grèce continentale sont moins vigoureuses et/ou plus instables. Les provenances de Grèce orientale sont instables et peu vigoureuses.

Lorsqu'on corrèle les performances de chaque provenance avec quelques caractéristiques écologiques de l'aire naturelle, il apparaît que les provenances qui manifestent la meilleure croissance en hauteur correspondent aux régions les plus arrosées, les moins arides (au sens de De Martonne), et situées sur la façade occidentale du pays. Le sapin de Céphalonie est certes une espèce pionnière, et résistante à la sécheresse; il n'en reste pas moins que ses exigences hydriques sont relativement élevées (bioclimats humide et sub-humide). Les résultats de mortalité et la diminution des pousses annuelles sont là pour en témoigner. Visiblement, à l'âge où les mesures ont été réalisées, les provenances originaires des régions les moins arrosées (Grèce orientale) ne conviennent pas au type de climat existant en plantations expérimentales (étage du chêne pubescent), bien qu'elles puissent présenter en théorie le meilleur potentiel adaptatif, face à des sécheresses estivales plus prononcées. Elles pourraient sans doute être utilisées pour des introductions à très basse altitude.

Ces résultats indiquent que des prospections de nouvelles ressources génétiques devraient être envisagées (Péloponnèse en tout premier lieu) pour échantillonner au mieux la variabilité de l'espèce, et augmenter ses possibilités d'amélioration. Un programme de sélection intra-provenance semble tout à fait envisageable, à condition que les estimations des paramètres génétiques réalisées ici 11 années après la graine soient comparables à celles qui pourront être faites à l'âge adulte. Étant donné l'adaptation de chacun des 3 groupes de provenances à des conditions écologiques particulière, il pourrait être aussi intéressant d'envisager une sélection faisant intervenir des croisements contrôlés inter-provenances.

\section{RÉFÉRENCES}

Baradat P (1976) Use of juvenile-mature relationships and information from relatives in combined multitrait selection. Proc IUFRO joint meet on Adv Generation Breeding, Bordeaux, France, 121-138

Baradat $P$ (1989) Amélioration génétique des arbres forestiers : éléments méthodologiques. Rapport INRA, Laboratoire d'Amélioration des Arbres Forestiers, Bordeaux

Barbero M, Quezel P (1976) Les groupements forestiers de Grèce Centro-Méridionale. Ecol Mediterr 2, 3-86

Barbero M, Quezel P (1985) Carte de la végétation potentielle de la région méditerranéenne, feuille $n^{\circ} 1$ : Méditerranée orientale. Éditions du CNRS, Paris 
Bassiotis C (1956) Fir forests in Greece. Ann Inst Sylvic Univ Thessalonique, 1-89

Bornovas J, Rondogianni-Tsiambaou T (1983) Geological map of Greece (1/500 000), Athens, 2nd edition

Burdon RD, Sweet GB (1976) Problems of interpreting inherent differences in tree growth shortly after planting. Tree Physiology and Yield Improvement (MGR Cannell, FT Last, eds). Academic Press, Londres, 483-502

Descroix $L$ (1981) Variabilité génétique du Sapin de Grèce et du Sapin de Nordmann. Étude comparée de leur comportement avec le Cèdre de l'Atlas. Mémoire l'ENITEF, INRA, laboratoire d'Amélioration des Arbres Forestiers, Bordeaux

Fady B (1988) Croissance du Sapin de Grèce : variabilité inter-provenances dans trois stations méditerranéennes françaises. Ann $\mathrm{Sci}$ For 45, 239-254

Fady B (1990a) Variabilité génétique du Sapin de Grèce. Thèse de Doctorat en Sciences, Université d'Aix-Marseille 3

Fady $B$ (1990b) Variabilité génétique des composantes de la croissance en hauteur du Sapin de Céphalonie (Abies cephalonica Loud). Can J For Res 20, 1453-1460

Fady B, Arbez M, Ferrandes $P$ (1991) Variability of juvenile Greek firs (Abies cephalonica Loud) and stability of characteristics with age. Silvae Genet (sous presse)

Falconer DS (1974) Introduction à la génétique quantitative. Masson, Paris

Mattfeld J (1930) Über hybridogene Sippen der Tannen. Nachgewiesen an den formen der Balkanhalbinsel. Bibliotheca Botanica 100, 184
Mitsopoulos DJ, Panetsos CP (1987) Origin of variation in fir forests in Greece. Silvae Genet 36, 1-15

Mode CJ, Robinson HF (1959) Pleiotropism and the genetic variance and covariance. Biometrics 15, 518-537

Moreau MC (1989) Contribution à l'étude de la structure génétique d'un peuplement de Sapin pectiné (Abies alba Mill) des BassesVosges. DEA d'Écologie Expérimentale, Université de Pau et des Pays de l'Adour

Namkoong G, Usanis RA, Silen RR (1972) Age related variation in genetic control of height growth in Douglas fir. Theor Appl Genet 42, 151-159

Namkoong G, Conkle MT (1976) Time trends in genetic control of height growth in ponderosa pine. For Sci 22, 2-13

Panetsos CP (1975) Monograph of Abies cephaIonica Loudon. Ann For 7, 1-22

Pauly D (1962) Aperçu sur l'écologie du sapin de Céphalonie et de ses hybrides. RFF 8-9, 755-769

Portefaix C (1989) Exploration de la variabilité génétique présente dans cinq peuplements naturels de pin Laricio de Corse (Pinus nigra ssp laricio var corsicana Loud). Ann Sci For 46, 217-232

Shea KL (1990) Genetic variation between and within populations of Engelmann spruce and subalpine fir. Genome 33, 1-8

Wricke G (1962) Uber eine Methode zur Erfassung des Okologische Strubreite in Feldversuchen. $Z$ Pflanzenzücht 47, 92-96

Wright JW (1976) Introduction to Forest Genetics. Academic Press, New York 